\title{
Environmentally-induced alternative oncogenesis: EROS arrows
}

\author{
Sergey K. Pinaev ${ }^{1 *}$, Olga G. Pinaeva ${ }^{1}$, and Alexey Ya. Chizhov ${ }^{2}$ \\ ${ }^{1}$ Far Eastern State Medical University, Khabarovsk, Russia \\ ${ }^{2}$ Peoples' Friendship University of Russia (RUDN University), Faculty of Ecology, 6 Miklukho- \\ Maklaya St, Moscow, 117198, Russian Federation
}

\begin{abstract}
The cause of the oncological diseases in children and the specific spectrum of this pathology, with hemangiomas prevailing among benign tumors (BT), and leukemia, lymphomas, and neural tissue tumors among malignant neoplasms (MN), remains unknown. The authors studied the similarities and differences of the connections between environmental factors, benign tumors and MN, as well as the relations between BT and $\mathrm{MN}$. The causality of the so-called "sporadic" fluctuations in the incidence of neoplasms and their relations with environmental factors were revealed. A hypothesis of environmentally-induced alternative oncogenesis is suggested. According to the hypothesis, fetal hemoglobin enhances environmentally-related oxidative stress (EROS), leading to epigenomic regulation disorder of SEMA 7A and MICAL family proteins in the endothelium, axon growth cone, and hematopoietic stem cells.
\end{abstract}

\section{Introduction}

The role of environmental factors in oncogenesis does not cause any doubts [1]. Moreover, the mechanism of development of many forms of tumors is still unclear. Thus, until now, the cause of the oncological diseases in children and the special spectrum of this pathology with the predominance of hemangiomas among benign tumors (BT), and leukemia, lymphomas, and nervous tissue tumors among malignant neoplasms (MN), remains unknown. While in adults the majority of cancer forms are caused by a prolonged exposure to specific organs and systems with a fairly well-known etiopathogenesis, oncological pathology occurs in children, as a rule, in the absence of signs of obvious oncogenic causes. Therefore, detecting the causes and ways of developing neoplasms in children is a very urgent objective aimed at working out measures for their prevention.

Objective of the study was to suggest a hypothesis of environmentally-induced oncogenesis in children.

\footnotetext{
* Corresponding author: pinaev@ mail.ru
} 


\section{Materials and methods:}

Our approach consists in analyzing the similarities and differences in the connections of environmental factors with benign and malignant neoplasias, as well as in the study of the relationship between BT and MN. To study the ecosystem, a system-determined dynamic balance model of the block type was worked out (Fig. 1). Balance dynamic models make it possible to evaluate the phenomena in their development as a set of processes of transfer of matter and energy, and the functional nature of the model allow extrapolating the revealed patterns and predict the future states of system objects on their base.

The incidence of BT in the population of young children (YC) 0-4 years old in the population of Khabarovsk was studied; 11 cohorts of children born in 1976-1986 were formed. The authors also studied the incidence of MN among YC in the Khabarovsk Region; 17 cohorts of the children born in 1972-1988 were formed .Using the IBM SPSS Statistics 23 software package, a regression-correlation analysis was performed between the dynamic series of the BT and $\mathrm{MN}$ incidence in cohorts using the time lag in relation to the year of birth in children with BT in seven iterations $(0,-1,-2,-3$ and $+1,+2,+3$ years). The results are interpreted based on an analytical review of publications in the PubMed, eLibrary, MEDLINE databases.

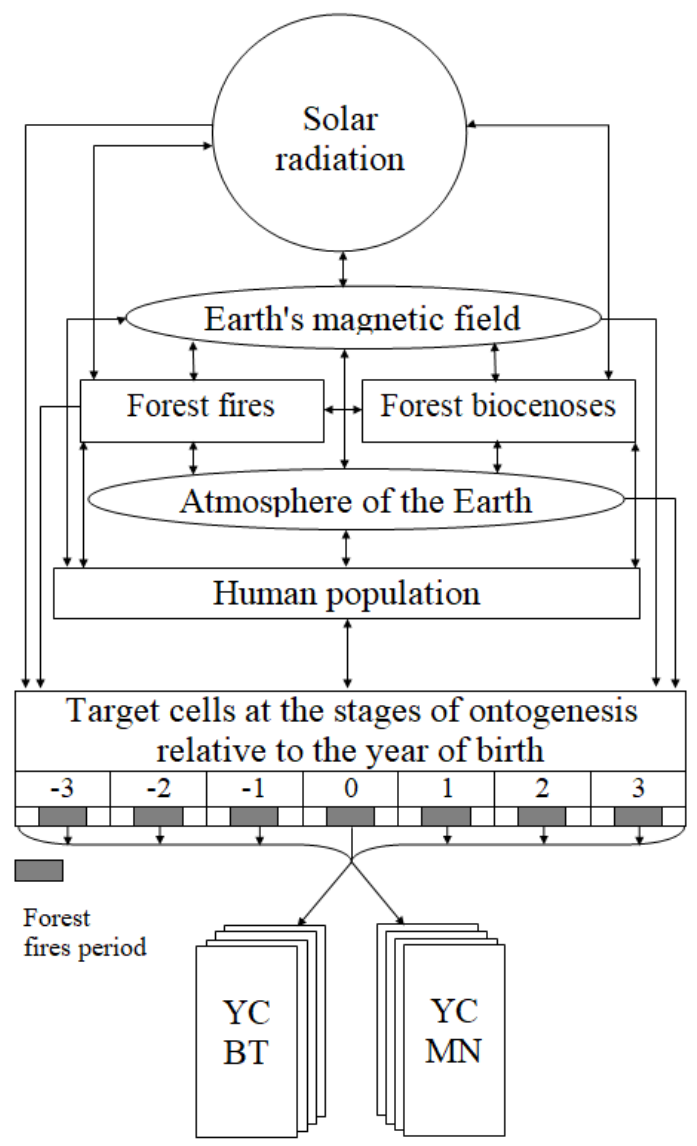

Fig. 1. The system-determined dynamic balance model of the ecosystem of the block type "Sun smoke of forest fires - YC population" 


\section{Results and discussion}

The relations between the incidence of hemangiomas and non-Hodgkin's lymphomas in cohorts 2 years older $(\mathrm{r}=0.769, \mathrm{p}=0.003)$ confirmed the previously identified lag difference between the activity of the Sun, the occurrence of hemangiomas ( -3 years) [2] and non-Hodgkin's lymphomas (-1 year) [3]. On the other hand, the correlation between the incidence of hemangiomas and leukemia $(r=0.675, \mathrm{p}=0.023)$ at a lag of +2 years reflects the relations of the former with postnatal exposure to smoke, while the incidence of leukemia confirms its preconceptive effect $[2,4]$. Generally, the negative correlation $(\mathrm{r}=-$ $0.705 ; p=0.015$ ) between the incidence of BT and $\mathrm{MN}$ in cohorts a year older [2] reflects the fact that the former's respond to solar activity in earlier periods of ontogenesis than the latters (Fig. 2).

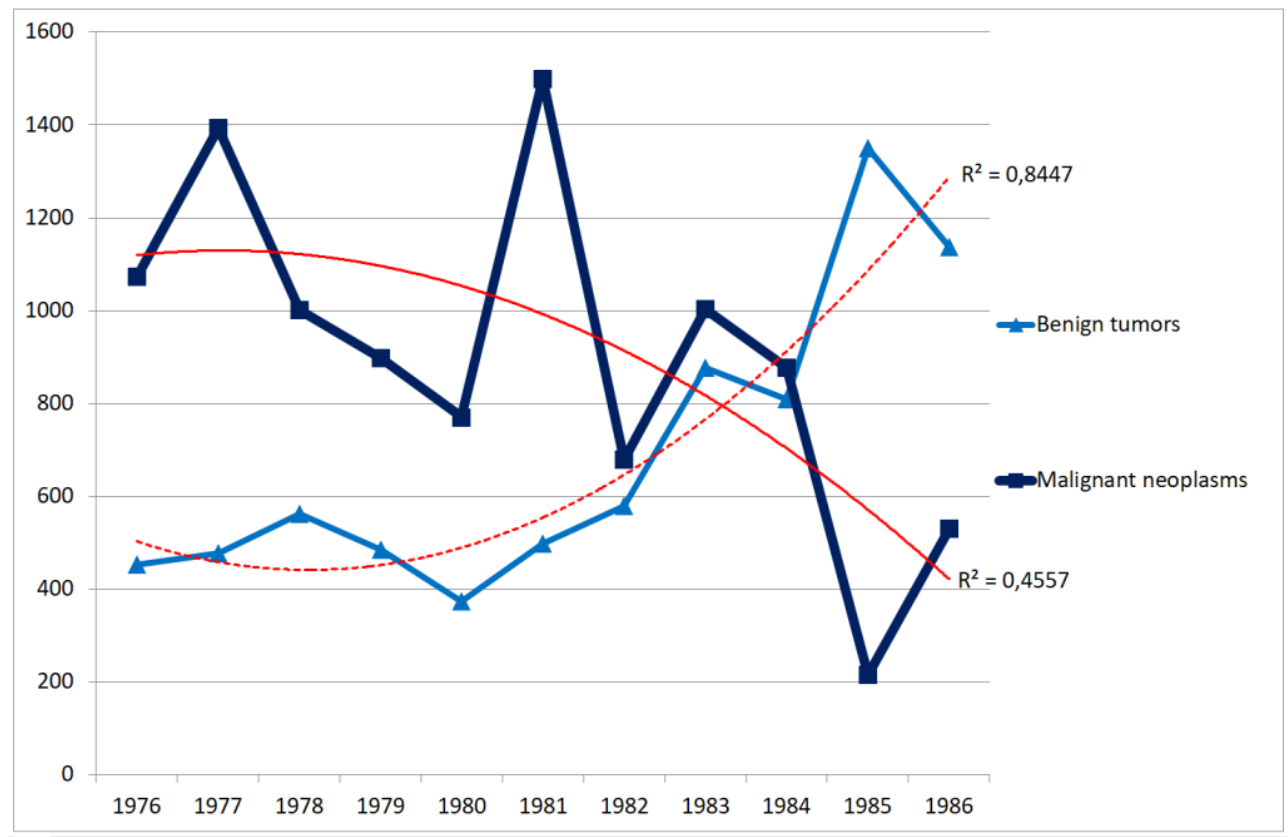

Fig. 2. BT incidence in the cohorts of $\mathrm{YC}\left(\right.$ per $\left.10^{5}\right)$ and $\mathrm{MN}\left(\right.$ per $\left.10^{6}\right)$, lag (-1) year.

\section{Conclusions}

Thus, the environmental factors connections with the incidence of benign tumors and $\mathrm{MN}$ have significant differences. For example, the preconceptive effect of solar activity is important for hemangiomas, and postnatal for leukemia. On the contrary, the smoke of forest fires affects the incidence of leukemia being exposed to ontogenesis during the preconceptive period, while for the incidence of hemangiomas, a connection with its postnatal exposure has been revealed $[2,4]$. On the whole, the data obtained by us allow stating the causality of the so-called "sporadic" fluctuations of neoplasms incidence and their connections with environmental factors.

We suggest a hypothesis of environmentally-induced alternative oncogenesis. According to it, the common vector of ecological factors is environmentally-related oxidative stress (EROS)[5]. 
Its results at the preconceptive stage of ontogenesis are epigenomic regulation disorders of semaphorins, especially SEMA 7A, and the MICAL family proteins. At the stage of placental period, the sensitive targets for EROS in the embryo and fetus are the embryonic and fetal hemoglobins, which trigger, due to their instability, the amplification of it [6].

An additional increase of EROS occurs in the mitochondria of all actively proliferating cells, especially in the growth cone of axon, bone marrow, and endothelium, where semaphorins are presented. Normally, semaphorins provide axonal guidance, angiogenesis, and proliferation of immune cells. If violations occur, the transition of physiological proliferation into tumor is possible [7]. The proteins of the MICAL family represent a direct path to oncogenesis by EROS, growth factors, and oncogenes [8].

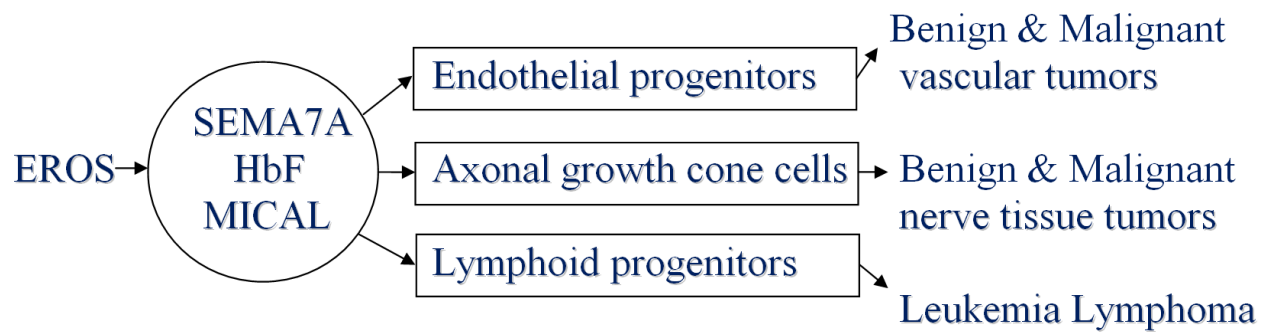

Fig. 3. "EROS arrows": Scheme of environmentally-induced alternative oncogenesis in YC.

Thus, environmentally-induced alternative oncogenesis is a consequence of amplification of EROS by fetal hemoglobin. This leads to epigenomic violations in the functions of semaphorins and MICAL in endothelial cells, bone marrow and lymphoid tissues directly contacting with red blood cells, and in the cells of the axon growth cone and its microenvironment [6].

The result of environmentally-induced alternative oncogenesis is a special spectrum of childhood neoplasms, with hemangiomas and lymphangiomas prevailing among BT, and hemoblastoses and neurogenic tumors among MN (Fig. 3). Moreover, depending on the changes in the parameters of the complex of environmental factors, certain forms of neoplasms arise predominantly during certain periods of time, forming alternative fluctuations of their incidence.

\section{References}

1. N.A. Agadzhanyan, A. Ya. Chischov, T.A. Kim, CIVILIZATION DISEASES. Human Ecology, 4, 8-11 (2003)

2. S.K. Pinaev, A.Ya. Chizhov, Advances in molecular oncology, 5(4), Appendix, P.18$19(2018)$

3. S.K. Pinaev, A.Ya. Chizhov, O.G. Pinaeva, Fundamental science and technology promising developments XVII, 2, 14-17(Academic, North Charleston, 2018)

4. A.Ya. Chizhov, S.K. Pinaev, Radiation and risk, 27(4), 87-94. (2018)

5. A.Ya. Chizhov, S.K. Pinaev, S.Z. Savin, Technologies of Living Systems, 1(9), 47-53 (2012)

6. J. S. Fossen, Methemoglobinemia: Infants at risk. Curr. Probl. Pediatr. Adolesc. Health Care, 49(3), 57-67 (2019)

7. G. Neufeld, Y. Mumblat, T. Smolkin, S. Toledano, I. Nir-Zvi, K. Ziv, O. Kessler, Cell Adh Migr, 10(6), 652-674 (2016)

8. Yoon J., Terman J.R., Mol Cell Oncol, 5(1), e1384881 